\title{
Correction to: Energy and Environmental Security in Developing Countries
}

\author{
Muhammad Asif
}

\section{Correction to:}

M. Asif (ed.),

Energy and Environmental Security in Developing Countries, Advanced Sciences and Technologies for Security Applications, https://doi.org/10.1007/978-3-030-63654-8

The original version of this book was inadvertently published with incorrect country name and without Dedication, Foreword, Preface, and Acknowledgement pages. These have now been updated.

Dedication, Foreword, Preface, and Acknowledgement pages have been included in the book front-matter and the country name has been updated from UK to Kingdom of Saudi Arabia. 